\title{
IX,
}

\section{Die centrifugale Leitung im sensiblen Endnearon.}

Von

Dr. Osear Kohnstamm (Königstein i. Taun.).

Im Jahre 1876 hat Stricker ${ }^{21}$ ) die principiell neue Thatsache entdeckt, dass ein an hinteren Wurzeln angebrachter Reiz eine centrifugalwärts fortschreitende Erregung erzeugt. Er sah nach elektrischer oder mechanischer Reizung des peripheren Stumpfes durchschnittener hinterer Lumbalwurzeln Gefässerweiterung im gleichseitigen Hinterbein. Steinach und Wiener ${ }^{18,19}$ ) fanden unter denselben Bedingungen Bewegungen von Darm und Blase beim Froseh. Um diese Reizerfolge zu erklären, nahm man an, dass die hintere Wurzel eine gewisse Auzahl von Nervenfasern führe, deren Ursprungszellen im Rückenmark gelegen seien. Denn die Identität der (cellulifugalen) Axonund der Leitungsrichtung gilt bis jetzt als Axiom. In der That hatten Kutschin, Freud, Klausner bei Petromyzon und Proteus, von Lenhossék, Ramón y Cajal, Retzius beim Hûhnchen derartige Elemente beschrieben. Es giebt noch eine Reihe anderer gewichtiger, aber wenig beachteter Thatsachen, die mir zu beweisen schienen, dass in den hinteren Wurzeln auch der Säugethiere centrifugale Erregungen fliessen. Nun hat man aber nach Verletzung des Rückenmarks oder Durchschneidung hinterer Wurzeln mit der so empfindlichen Marchimethode keine centrifugalen Markfasern in denselben finden können (Singer-Münzer, Münzer-Wiener, Verfasser, Bayliss). Andererseits degeneriren nach Durchschneidung hinterer Wurzeln sämmtliche Fasern des centralen Stumpfes (Sherrington ${ }^{17}$ )). Schliesslich erklären Fread und Sherrington die bei niederen Wirbelthieren beobachteten intrespinalen Ursprungszellen hinterer Wurzeln mit gutem Grund für Spinalganglienzellen, die ins Centralorgan gewissermassen dislocirt seien, ein Verhalten, das bei Amphioxus und Petromyzon noch deutlich zu erkennen ist.

So kam ich dazu, die Hypothese aufzustellen, „dass die centripetalen Neurone rom Centralorgan aus erregt werden können. Mir ist keine Thatsache bekannt, die eine solche Vorstellung ausschlösse" (Verf. 13). Ich wies damit auf die Möglichkeit hin, dass die Träger der centrifugalen Leitung in den hinteren Wurzeln identisch seien mit den centripetalen Neuronen, die in den Spinalganglien wurzeln. Als Experimentum crucis 
schlug ich den Versuch vor, den selbst anzustellen mir nicht vergönnt war, die peripheren Stümpfe der hinteren Wurzeln erst dann zu reizen, „nachdem man vorher mittelst Durchschneidung der hinteren Wurzeln die hypothetischen endogenen Neurone zur Degeneration gebracht hätte". Bliebe*) der Reizerfolg positiv, wie unmittelbar nach der Durchschneidung, so sei damit die Identität der centrifugalen Bahn mit dem sensiblen Endneuron erwiesen.

Wenige Monate nach dieser Publication hat in der That Bayliss ${ }^{2}$ ) auf dem von mir angedeuteten Wege den erwarteten Nachweis erbracht, dass die gefässerweiternde Wirkung der Reizung hinterer Wurzeln beim Hund an Neurone gebunden sei, die in den Spinalganglien wurzeln. Denn der Reizerfolg bestand fort, wenn 10 Tage vor der Reizung die hinteren Wurzeln durchschnitten worden waren; er fehlte aber, wenn distal vom Ganglion durchschnitten und der distale Stumpf 10 Tage später gereizt wurde. Damit war die fundamentale Thatsache erwiesen, dass das sensible Endneuron nicht nurLeiter der Sensibilität ist, sondern auch centrifugalen, oder, wie Bayliss sagt, antidromen Erregungen dient. Die vasoconstrictorische Innervation scheint mehr an das System der vorderen Wurzeln und des Sympathicus gebunden zu sein. Ein weiteres wesentliches Argument für die Identität der sensiblen und der vasodilatatorischen Leitung liegt in dem von Bayliss gelieferten Nachweis, dass der Sitz der durch Hinterwurzelreizung ausgelösten Gefässerweiterung im Wesentlichen die Haut und nur in viel geringerem Maasse die Musculatur sei.

Wie der peripherische Erfolg der auf dem sensiblen Endneuron ablaufenden centrifugalen Innervation zu Stande kommt, darüber können wir uns folgende Vorstellung machen: Das sensible Endorgan kann und muss, ebenso wie es durch Hautreize erregt wird, auch von seinem Nerven aus, gleichermassen an seinem centralen Pol, erregt werden können. Die Erregung des Endorgans löst dann vasomotorische Reflexe aus. Solche Reflexe brauchen das Centralorgan nicht zu passiren. Wir wissen ja, wie schnell und leicht vasomotorische Reflexe der Haut bei Temperaturreizen und bei der Urticaria factitia zu Stande kommen. Die strenge Localisation an den Ort des Reizes macht es für diese Vorgänge sehr wahrscheinlich, dass sie auf kurzen peripherischen Reflexbögen ablaufen. Besonders Jeicht müssen solche Uebertragungen in den sensiblen Endplexus vor sich gehen; nur kann man nicht sagen, ob und welche gangliösen Elemente dafür in Betracht kommen. Zu Genüge nachgewiesen sind periphere Reflexbögen vielfach im Bereich des Bauchsympathicus.

*) Im Text heisst es in Folge Druchfehlers ,ausbleiben" statt „, bleiben“" "3). 
Die von mir entwickelte Vorstellung ${ }^{13}$ ) über das Zustandekommen des Stricker'schen Phänomens erinnert an die Axonreflexe Langley's, wie auch Bayliss bemerkt.

Abgèsehen von dem peripherischen Erfolg der Hinterwurzelreizung bei dem Fehlen von centrifugal gerichteten Hinterwurzelneuronen bin ich zu meiner Hypothese von der centrifugalen oder antidromen Leitung im sensiblen Endneuron durch einige Deductionen geführt worden, die einer näheren Besprechung bedürfen.

Sie betreffen:

1) das Phänomen des Reflexes von hinterer Wurzel auf hintere Wurzel,

2) die trophischen Störungen, die im Thierversuch, bei organischen Nervenkrankheiten, bei den vasomotorisch-trophischen Neurosen beobachtet werden,

3) die Pathologie des Herpes zoster,

4) die rückläufigen Systeme innerhalb sensibler Neuroncomplexe des Centralnervensystems,

5) die Tigrolyse der Spinalganglienzelle nach Durchschneidung ihres peripheren Fortsatzes,

6) das aus dem Vorausgegangenen erschlossene doppelsinnige Leitungsvermögen der Spinalganglienzelle.

1) Gotch und Horsley ${ }^{6}$ ) hatten an Säugethieren, sowie später Mislawsky ${ }^{16}$ ) am Frosch nachgewiesen, dass eine auf dem Weg hinterer Wurzeln ins Rückenmark eintretende Erregung am centralen Querschnitt anderer hinterer Wurzeln als negative Schwankung wieder zu Tage trete. Dies elektrische Phänomen konnte durch elektrische Reizung des Ischiadicus, aber auch durch adäquate Reizung, nämlich durch Kneifen der Zehen ausgelöst werden. Die aus dem Rückenmark austretende Erregung durchsetzt die Spinalganglien. Denn die negative Schwankung konnte auch am Ischiadicus nachgewiesen werden, dessen motorische Elemente durch rechtzeitig vorausgegangene Durchschneidung und Degeneration der zugehörigen vorderen Wurzeln ausgeschaltet waren. Mislawsky fasst die Erscheinung als wirklichen Reflex auf, dessen ableitender Schenkel durch centrifugale Hinterwurzelfasern gebildet würde. Dabei stützt er sich besonders auf die Untersuchungen Steinach's, der durch Reizung hinterer Wurzeln vom Frosch Darmbewegungen ausgelöst und damit die Existenz centrifugaler Hinterwurzelfasern darzuthun geglaubt hat.

Dem gegenüber wies ich darauf hin, dass der "Reflex von hinterer Wurzel auf hintere Wurzel" von Goteh und Horsley auch bei Sängethieren beobachtet worden sei, die nach anderer und meinen eigenen $\mathrm{Be}$ obachtungen centrifugaler Hinterwurzelfasern ermangeln. Unter diesen 
Umständen hielt ich es nicht für zulässig, den „Reflex von hinterer Wurzel auf hintere Wurzel" auf centrifugale Hinterwurzelfasern zu beziehen, und wies ausser auf eine gewisse Analogie mit dem Hering'schen Phänomen*) ${ }^{9}$ ) auf die Möglichheit hin, dass die Endverästelungen des gereizten Neurones, bezw. seiner Collateralen in der grauen Substanz direct oder indirect auf die Endverästelungen derjenigen Neurone einwirkten, deren negative Schwankung an der hinteren Wurzel zur Beobachtung kommt. Offenbar auf Grund desselben Mechanismus treten, wie Gotch und Horsley zeigen, bei Strychninvergiftung oder bei Reizung des Rückenmarksquerschnitts Actionsströme in die hinteren Wurzeln ein. Von allen Erregungen, die das Rückenmark durchlaufen, scheinen also diese in Mitleidenschaft gezogen zu werden.

Schon diese Versuche lebren eine centrifugale Leitung im sensiblen Endneuron. Das doppelsinnige Leitungsvermögen peripherer Neurone ist zwar längst erwiesen. Dass es auch dem sensiblen Endzeuron trotz der Zwischenschaltung der Spinalganglienzelle zukommt, kann nun aus Versuchen, wie denen von E. Du Bois-Reymond, GotchHorsley, Steinach ${ }^{20}$ ) u. A., abgeleitet werden.

2) Seitdem Mayo und Magendie zuerst die neuroparalytische Hornhautentzündung nach Trigeminus-Durchschneidung beschrieben hatten, wurde es klar, das die Ernährung der Gewebe in irgend einer Weise vom Nervensystem abhängig sei. Bekanntlich wurde und wird bis auf den heutigen Tag jene Augenerkrankung von den Einen als die Wirkung der Durchtrennung trophischer Fasern, von den Anderen als blosse Folge der Gefühllosigkeit der Gewebe aufgefasst. Die meisten Vertreter der letzteren Annahme, sei es nun, dass sie mehr an allgemeine Insulte denken, oder dass sie speciell die Austrocknung der Hornhaut anschuldigen, die durch das Ausbleiben des sonst reflectorisch angeregten Lidschlages zu Stande krommt, sehen sich genöthigt, eine verminderte Widerstandsfähigkeit der Gewebe anzunehmen. Indem sie aber eine solche Wirkung der Nervendurchschneidung statuiren, nähern sie sich schon der Annahme einer besonderen trophischen Function.

Derselbe Parallelismus zwischen sensiblen, trophischen und, wie hier hinzugefügt sei, vasomotorischen Störungen tritt in einer grossen Anzahl von Nervenkrankheiten hervor. Als Vertreter der organischen Nervenkrankheiten diene - neben der tabischen Arthropathie u. a.m. die Syringomyelie, deren sensibles Hauptsymptom die partielle

*) Durch den Actionsstrom hervorgerufene Nervenerregung, die in unserem Fall an der Schnittstelle des Rückenmarks stattfinden könnte. Dieses ist in den betreffenden Versuchen central vom Versuchsfeld durchschnitten. 
Empfindungslähmung (des Schmerz- und Temperatursinnes), durch Zerstörung derjenigen Stellen in den hinteren und mittleren Theilen der grauen Substanz bedingt ist, wo das sensible Endneuron seine Erregung auf das zweite kreuzende Neuron überträgt (vergl. Verf., 11). Hier muss auch der Ort oder einer von den Orten sein, wo das sensible Endneuron seine centrifugalen Erregungen empfängt. Unter den vasomotorisch-trophischen Neurosen, für deren Auffassung die von uns entwickelte Anschauung besondere Wichtigkeit gewinnen dürfte, verweise ich auf die Erythromelagie und ihre erschöpfende Schilderung: in dem ausgezeichneten Werk von Cassirer ${ }^{5}$ ). Die Hauptsymptome, Hautröthung und Schmerz,stellenReizungszuständeder vasodilatatorischen und der sensiblen Innervation dar. In dem Sectionsfall Auerbach's ${ }^{1}$ ) fand sich eine Erkrankung hinterer Wurzeln. - Als Substrat trophischer Hautstörung findet sich nach Cassirer bäufiger ein Reizzustand als ein Ausfall der Nervenbahn.

Trophische und vasodilatatorische Innervation stehen in sehr enger Beziehung zu einander, besonders wenn man den wahrscheinlichen Einfluss des Nervensystems auf die Durchlässigkeit der Capillaren bedenkt. Sollte auch die trophische Innervation in den sensiblen Bahnen fliessen, so ergäbe sich von selbst die Einheitlichkeit der trophischen und vasodilatatorischen Leitungswege. Für den unzweifelhaften Thatbestand, dass es trophische Einflüsse giebt, die sich längs der peripherisch-sensiblen Bahnen fortpflanzen, ist stets nur diese Alternative formulirt worden: Tritt die trophische Störung der Hautgebilde ein, weil die centripetale Leitung unterbrochen ist, oder verlaufen mit den sensiblen Nerven centrifugale trophische Fasern? Die dritte Möglichkeit, dass die sensiblen Endneurone gleichzeitig der centrifugalen Leitung trophischer Impulse dienen, ist - soviel ich sehe früher von Niemand als gelegentlich von Weir-Mitchell*) in Betracht gezogen worden, und doch ist sie die nächstliegende, wenn man sich über die in der gewöhnlichen Fassung des Bellschen Gesetzes liegende Ausschliesslichkeit hinwegsetzt und sich von dem doppelsinnigen Leitungsvermögen des sensiblen Endneurons überzeugt hat.

3) Von der grössten Bedeutung für unsere Frage aber sind die Erfahrungen über die Pathologie der Gürtelrose. Nachdem zum ersten Mal im Jahre 1861 v. Bärensprung einen Zusammenhang zwischen der Erkrankung eines Spinalganglions und dem Herpes zoster erkannt hatte, der von allen späteren Autoren bestätigt wurde, ist vor Kurzem die glänzende Arbeit ron Head und Campbell erschienen, die sich

*) Vergl. Cassirer5) S. 53 
auf 450 Beobachtungen an Lebenden und auf 21 genane makro- und mikroskopische Autopsien stützt ${ }^{8}$ ).

Die Topographie des Ausschlags ist nach diesen Forschungen nicht durch das Ausbreitungsgebiet der peripheren Nerven, sondern durch die segmentalen Hautzonen bestimmt. Der Sitz der Erkrankung müsste demach entweder im Centralorgan oder im Ganglion gelegen sein. Während aber ersteres im Allgemeinen, abgesehen von der secundären Degeneration der erkrankten sensiblen Endneurone nichts Krankhaftes zeigt, erweist sich das Ganglion als Sitz schwerer entzündlicher Processe theilweise hämorrhagischer Natur, die zur Zerstörung einer Anzahl ron Nervenzellen geführt haben. Head und Campbell erklären danach die Gürtelrose für eine specifische Erkrankung der sensiblen Endneurone, ähnlich wie die Poliomyelitis anterior eine Erkrankung der motorischen Endneurone sei. Ueber den Zusammenhang zwischen der Erkrankung von Ganglion und Haut lesen wir nur die vorsichtige Aeusserung (a. a. 0.): „We do not imagine, that the eruption of herpes zoster is produced by disturbance of special trophic nerves, but by intense irritation of cells in the ganglion, which normally subserve the function of pain ..."

Ein Zusammenhang lässt sich in versehiedener Art denken. 1. könnte die Unterbrechung der centripetalen Leitung angeschuldigt werden. Dann müsste auch einfache Nervendurchschneidung Aehnliches bewirken, was nicht der Fall ist, oder 2. die Haut könnte erkranken, sobald die secundäre Degeneration der lädirten Neurone dieselbe erreichte. Die Erkrankung tritt aber schon auf, ehe eine Spur von Marchi-Degeneration nachgewiesen werden kann, wie gleich näher gezeigt wird. Oder schliesslich 3. die Zellerkrankung übt einen auf der Bahn der Nervenfortsätze weiter geleiteten echten Nerveureiz aus, ron einer Stärke, einer Qualität und einem Rhythmus, dass er den eigenthümlichen Krankheitszustand hervorruft. In diesem Fall müsste der Herpes ungefähr gleichzeitig mit der Entzündung des Ganglions auftreten. -

Das Material von Head und Campbell scheint in seiner seltenen Vollständigkeit wie aus einem Experiment ein eindeutiges Urtheil in dieser Richtung zu gestatten, obgleich die Autoren selbst über die Frage schweigen.

Bekanntlich tritt Marchidegeneration in voller Ausbildung ungefähr am 12. Tage nach Verletzung eines Neurons auf, während ich sie im Thierexperiment noch am 9. Tage vermisst habe. Also darf, wenn Zell- und Hauterkrankung gleichzeitig eingesetzt haben soll, Marchidenegeration nicht früher, als ungefähr 12 Tage nach Auftreten des Herpes nachzuweisen sein. In der That ist in den beiden Fällen, 
die 3 bezw. 9 Tage nach dem ersten Beginn des Herpes starben, keine Spur von Marchidegeneration nachgewiesen worden, eine vollständig ausgebildete aber in dem Falle, der nach 12 Tagen zufällig zur Section kam. Daraus dürfte in der That ziemlich streng hervorgehen, dass Zell- und Hauterkrankung ungefähr gleichzeitig stattgefunden haben. Ein Incubationsstadium kann trotzdem sehr wohl vorausgehen, ohne dass während desselben schon eine Zerstörung im Ganglion Platz greifen müsste.

Aus der Pathologie des Gürtelrose geht also hervor, dass eine eigenartige Reizung des sensiblen Endneurons eine krankhafte Veränderung auf der Haut hervorzubringen vermag. Die Erregung kann vom Spinalganglion aus auf zweierlei Wegen die Haut erreichen. Einmal könnte sie im Rückenmark oder Sympathicus Reflexe trophischer Natur auslösen, die einen gebahnten Weg zu der dem Spinalganglion entsprechenden Hautzone vorraussetzten. Diese unwahrscheinliche Hülfsannahme ist nicht erforderlich, wenn man den Reiz direct im peripherischen Fortsatz der Spinalganglienzelle absteigen lässt, wobei er nirgends anders hingelangen kann, als eben nach dem Locus morbi in der Haut. Das ist die einfachere Erklärung und passt zu den übrigen Momenten, die uns von der centrifugalen Leitung im sensiblen Endneuron überzeugen. Für die trophische Bedentung des Spinalganglions spricht auch einigermassen die Beobachtung Claude Bernard's, dass die neuroparalytische Hornhautentzündung vermieden wird, wenn man den Trigeminus möglichst weit central vom Ganglion Gasseri durchschneidet und dadurch dieses vor dem Entzündungsreiz sichert. - Man darf wohl annehmen (s. o.), dass von allen den Erregungen, die das Rückenmarksgrau kreuz und quer durchziehen, ein Theil in die hinteren Wurzeln eintritt und der trophischen Innervation des Hautorgans dient. Wie für den Skeletmuskel und die Geschmacksknospe, die nach Durchschneidung des Glossopharyngeus degenerirt, so sind auch für die Haut die specifischen, d. h. die sensiblen Nerven gleichzeitig die Träger der Trophik. - Eine weitere Quelle der Erregungen für das sensible Endneuron sind wahrscheinlich die sympathischen Fasern, die nach Ehrlich, Ramón y Cajal und Dogiel gewisse Zellen der Spinalganglien umspinnen.

4. Wir kennen übrigens seit Jahren noch eine Einrichtung, die für centrifugale Leitung innerhalb sensibler Apparate spricht. Wenn wir nämlicb von der allgemeinen und sehr gut begründeten Annahme*) ausgehen, von der nur das sensible Endneuron ausgenommen wird,

*) Die durch die Golgi- und die Marchimethode aufgebaute Tektonik der langen Bahnen beruht auf dieser Annahme, die das eigentliche Wesen der Neuronlehre ausmacht. 
dass die (cellulifugale) Richtung des Axons die Leitungsrichtung des Neuron angjebt, so kann für das nach der Netzhaut gerichtete Fasersystem (Ramón y Cajal, Wallenberg ${ }^{22}$ ), die rückläufigen Neurone in der centralen Gehörleitung (Held), die im Nucleus cuneatus externus endigenden Kleinhirnfasern (A. Thomas) kein anderer Sinn gefunden werden, als dass sie der nervösen Einstellung der Sinmesflächen dienen, sei es nun, dass sie deren Erregbarkeit hemmen oder steigern. Zwar wird gelehrt, dass die erhöhte Anspruchsfähigkeit sensibler Apparate, die mit dem Bewusstseinszustand der Aufmerksamkeit einhergeht, auf einer Innervation centraler Instanzen beruht (Wundt's Apperception*)). Damit ist das Problem nur nach oben verschoben, aber nicht gelöst. Es ist gerade so gut denkbar und keinenfalls die Unmöglichkeit davon erwiesen, dass direct die peripheren Endapparate innervirt werden. Gerade die sensiblen Teleneurone, zu deren Endkernen der Nucleus cuneatus externus (s. o.) gehört, erscheinen mit ihrem doppelsinnigen Leitungsvermögen nunmehr geeignet, als Träger accomodativer Innervation der Hautsinnesfläche zu dienen.

Wenn so ein Weg plausibel zu machen wäre, auf dem mit Vorstellungen verbundene Innervationen sich bis zur Haut fortzupflanzen vermöchten, wenn mit anderen Worten der nervöse Verbindungsweg zwischen Empfindungskreis und corticaler Fühlsphäre auch in umgekehrter Richtung befahren werden könnte, so wären die gut beobachteten Fälle von Stigmatisirung Hysterischer und Hypnotisirter nicht mehr so absolut unbegreiflich, wie vorher.

5. Für eine Reihe von Zellarten ist nachgewiesen worden, dass sie unmittelbar nach Durchschneidung ihrer Nervenfortsätze eine regressive Zustandsänderung durchmachen, dje als Chromatolyse oder besser als reactive Tigrolyse bezeichnet wird. Dazu gehören

I. die Zellarten motorischer Structur im Sinne Nissl's, d. h. a) die Vorderwurzelzellen**) des Rückenmarks und Hirnstamms and b) die von mir sogenannten Coordinationszellen einschliesslich der Riesenpyramiden der höheren Säugethiere ${ }^{* * *}$ ),

II. die Zellen des dorsalen Vaguskerns und des mesencephalen Trigeminuskerns,

*) Vergl. auch S. Exner's ,Attentionelle Bahnung“ u. O. Kohnstamm's „Einstellungsbahnen". Pflüger's Archiv. Bd. 89. S. 247.

**) Die im Vorderhorn gelegenen motorischen Zellen 1. Ordnung und ihre Homologe im Hirnstamm nenne ich Vorderwurzelzellen im Gegensatz zu den in ihrer Nähe gelegenen, nach demselben Typus gebauten motorischen Zellen 2. Ordnung (,Coordinationszellen“), die nicht Vorderwurzelfasern, sondern Strangfasern ("Coordinationsbahnen") den Ursprung geben ${ }^{12}$ ).

***) Die entsprechenden Zellen der Hirnrinde vom Kaninchen ermangeln der "motorischen" Structur und zeigten - mir wenigstens - keine reactive 
III. die Zellen der Clarke'schen Säule,

IV. die Zellen der Spinalganglien und ihrer eranialen Homologa, sowie der sympathischen Ganglien.

Den Spinalganglien kommt das eigenartige Verhalten zn, dass ihre Tigrolyse nur nach Durchschneidung des peripherischen Fortsatzes eintritt, sehr wenig nach der des centralen, der hinteren Wurzel. Man hat sich - dem Neuronschema zu Liebe - gewöhnt, die hintere Wurzel dem Axon, den peripheren Nerven einem Dendriten zu homologisiren. Da aber die anderen Zellen gerade nach Durchschneidung der Axone in Tigrolyse gerathen, muss man im Lichte dieser neuen Erfahrung den peripherischen Fortsatz ebenfalls als Axon der Spinalganglienzelle ansprechen. Nachdem wir die centrifugale Strömung im sensiblen Endneuron kennen gelernt haben, kann auf alle Zellarten die Formel angewandt werden, dass sie in Tigrolyse gerathen, wenn infolge von Verletzung des Axons der Abfluss der Erregung gehemmt ist. - Das Intactbleiben der Zelle nach Durchschneidung der hinteren Wurzel erfordert die Hülfsannahme, dass die centripetale Erregungswelle nach Stärke und Form nicht geeignet ist, bei ihrer Rückstauung die Zelle zu schädigen. - Die meisten Autoren erklären im Gegensatz zu r. Lenhossék's und meiner eben dargelegten Auffassung die Tigrolyse der Spinalganglienzelle aus dem Zustand der Inactivität, in den sie gerathe, nachdem die zufliessenden Impulse abgeschnitten sind. Diese Erklärung würde auf keine andere Zellart stimmen und dann „sind z. B. die Clarke'schen Zellen nach Durchschneidung ihrer Axone, der Kleinhirnseitenstrangbahn, noch gerade so den zufliessenden Reizen zugänglich wie vorher, und andererseits haben (...) Macdonald und Reid (...) gezeigt, dass die durchschnittenen Zwerchfellnerven des durch künstliche Athmung am Leben erhaltenen Thieres beim Aussetzen derselben und Nachlassen der Apnoe stundenlang einen rhythmischen elektrischen Actionsstrom zeigten, entsprechend den bulbären Impulsen, die den durchschnittenen Nerven nach wie vor zufliessen" (Verf. ${ }^{10}$ ).

Goldscheider's beachtenswerther Hinweis, dass den Ursprungskernen der durch Nervendurchschneidung gelähmten Muskeln aus diesen keine

Tigrolyse. Trotzdem sind sie als Ursprungszellen der Pyramidenbahn Homologa der Riesenpyramidenzellen. Es giebt also Zellen motorischer Function ohne motorische Structur (vgl, auch die Zellen des dorsalen Vaguskerns). Keinenfalls hat Kolmer ${ }^{14}$ ) Recht in seinem Satz: „Für die vergleichend-anatomisehe Forschung ergiebt sieh die Thatsache, dass ganz unabhängig von der so viel umstrittenen mikroskopischen Configuration der Rinde in ihren einzelnen Bezirken und Windungen das übereinstimmende Auftreten bestimmt charikterisirter Zellen der geeignetste Anhaltspunkt für das Auffinden homologer Regionen der verschiedenen Thiere ist." 
centripetalen Erregungen mehr zukämen, passt nur für den Fall der Vorderwurzelzellen. Die Inactivitätstheorie besteht hingegen zu Recht für die langsam eintretenden retrograden und metaneuralen (d. h. über die Neurongrenze hinausgreifendien) Atrophien und Agenesien, wie sie experimentell durch die Bedingungen der G u d d en'schen Methode herbeigeführt werden.

6) Ebenso sicher, wie das doppelsinnige Leitungsvermögen der Nervenfaser bewiesen war, glaubte man von der Ganglienzelle behaupten zu dürfen, dass sie „dynamisch polarisirt" sei, d. h. dass sie Erregungen nur in einer Richtung, der des Axons, aus sich bervorgehen lasse. Das Beispiel der auch von centrifugalen Erregungen durchsetzten Spinalganglienzelle lehrt, das diese Regel keinesfalls für alle Gattungen von Nervenzellen gilt. Sie ist vielmehr nur für die Vorderwurzelzellen streng erwiesen und zwar durch das Bell'sche Gesetz: Reizung der hinteren Wurzel macht motorische Reflexe, die auch elektrisch als negative Schwankung am centralen Querschnitt vorderer Wurzeln nachweisbar sind. Centrale Reizung der durchschnittenen vorderen Wurzel bleibt ohne mechanischen oder elektrischen Erfolg an vorderen und hinteren Wurzeln. Die Vorderwurzelzelle lässt also Erregungen nur in der Richtung nach aussen durch, sie bildet einen Ventilverschluss, bloc der Engländer, für entgegengesetzt gerichtete Erregungen. In diesem übersichtlichen Falle versagt übrigens die Hypothese ron der physiologischen Bedeutung der Neurofibrillen, die nach Bethe's beweisenden Präparaten Dendrit und Neurit continuirlich durchziehen $^{3}$ ). Bei ihrer supponirten Function als einfache, nicht polarisirte Leiter müssten sie, ebenso gut wie cellulifugal, auch in der Richtung von Axon nach Dendrit leiten und die Auslösung von Reflexen auf Vorderwurzelreizung ermöglichen. Der Bell'sche Versuch lehrt, dass dies nicht der Fall ist, dass also die Leitungsfähigkeit in oder vor der Zelle unter der Herrschaft besonderer Bedingungen steht und eine absolute functionelle Continuität nicht existirt. In demselben Sinne spricht die zeitliche Verzögerung der Erregungsleitung beim Uebertritt centraler oder reflectorischer Erregungen auf das motorische Endneuron.

Da eine bei centraler Vorderwurzelreizung in die Zelle eintretende Frregung auf dem Dendriten keinen Ausweg findet, muss bei fortgesetzter Reizung von motorischen Nerven ein erhebliches Quantum von kinetischer Energie in oder jenseits der Zelle verschwinden. Vielleicht ist die unter solchen Bedingungen von Nissl beobachtete "Pyknomorphie" des Facialiskerns von diesem energetischen Gesichtspunkte aus zu verstehen.

Für alle anderen Zellarten ist bis jetzt eine gewisse Möglichkeit der doppelsinnigen Leitung nicht ausgeschlossen. Für die Spinalganglienzelle ist sie erwiesen. Man wende nicht ein, wienenerdings La n gen d o rff 15 ) 
und dann Steinach ${ }^{20}$ ) zu beweisen suchten, dass die Erregung den verticalen Schenkel des $T$ nicht benutze und an der Zelle vorbeifliesse!

Was soll aber dieser anders führen, als Fibrillen, die von den Fortsätzen zur Zelle und umgekehrt verlaufen! Im embryonalen Zustand und bei Fischen bildet die hier bipolare Zelle die einzige Verbindung zwischen beiden. Fortsätzen, und Bethe ${ }^{t}$ ) hat nur solche Fibrillen gesehen, die von beiden Fortsätzen in die Spinalganglienzelle eintreten*). Aus den interessanten Versuchen der vorgenannten Forscher ${ }^{15,20}$, die durch Nicotinvergiftung und durch Anämisirung das Spinalganglion abzutödten versuchten, geht meines Erachtens nur her. vor, dass dessen Zellen bezw. die leitenden Bestandtheile derselben widerstandsfähiger sind, als andere Nervenzellen, an denen ein Neuronübergang stattfindet. So verhindert die Nicotinisirung der sympathischen Ganglien (Langley) und die Anämisirung der Vorderwurzelzellen (Ehrlich und Brieger) den Uebergang der Erregung von einem Neuron zum anderen. Ebenso ist die motorische Nervenendplatte im Muskel der empfindlichste Theil des neuromusculären Apparates. An den mit dem Neuronübergang verbundenen dynamischen Bedingungen mag es auch liegen, dass bei dem Uebertritt der Erregung auf das motorische Endneuron eine Leitungsverzögerung statthat, die beim Durchsetzen des Spinalganglion nur unter bestimmten Versuchsbedingungen nachweisbar ist. Während Exner am Spinalganglion eine Verzögerung vermisste, bedingte die.Einschaltung des Ganglion jugulare inferius in den Versuchen von Gad und Joseph ${ }^{7}$ ) eine Verlängerung der Latenzzeit der Athmungsreflexe, die vergleichsweise durch Vagusreizung distal und central vom Ganglion ausgelöst wurden. Die von Steinach ${ }^{20}$ ) rorgefundenen histologischen Veränderungen an den Spinalganglienzellen beweisen übrigens nichts für deren Functionsunfähigkeit. Denn der schwer degenerative Zustand von Tigrolyse und Atrophie nach Nervendurchschneidungen und Amputationen fubrt nicht zu Waller'scher Degeneration der mit dem Spinalganglion verbunden gebliebenen Theile und hebt nicht die Leitungsfähigkeit durch das Ganglion auf. Die dem histologischen Anschein nach todte Zelle erfüllt noch ihre Aufgabe als trophisches Centrum des Neuron und als Leiter.

Da aber die für das sensible Endneuron erschlossene doppelsinnige Leitung die Spinalganglienzelle durchsetzt, darf folgender für die Physiologie der Nervenzelle wichtige Sehluss gezogen werden: Das doppelsinnige Leitungsvermögen ist für alle Arten von Nervenzellen in Betracht zu zieben und kann bis jetzt nur für die Vorderwurzelzellen streng ausgeschlossen werden.

*) Ebenso G. Mann, Anat. Anzeiger. Bd. 14. Ergänzungsbd. 


\section{Literatur.}

1) S. A u erbach, Ueber Erythromelalgie. D. Ztschr. t. Nervenheilk. 1897. XI, 1 u.2.

2) W. M. Bayliss, On the origin from the spinal cord of the vasodilator fibres etc. Journ. of physiology. 1901. 3 u. 4.

3) A. Bethe, Ueber die Primitivfibrillen in den Ganglienzellen etc. Schwalbe's morpholog. Arbeit. VIII, 1.

1) Derselbe, Ueber die Primitivfibrillen in den Ganglienzellen etc. Arch. f. mikroskop. Anat. 1900.

5) R. Cassirer, Die vasomotorisch-trophischen Neurosen. Berlin 1901.

6) Gotch and Horsley, On the mammalian nervous system ete. Phil. Transaction 1891.

7) J. Gad und M. Joseph, Ueber die Beziehungen der Nervenfasern zu den Nervenzellen in den Spinalganglien. Arch, f. Phys, 1899. 3 u. 4.

S) H. Head u. A. W. Campbell, The pathology of herpes zoster etc. Brain 1900.

9) E. Hering, Sitzb. d. k. Akad. d. Wiss. Wien. LXXXV. III. Abth. 1882.

10) O. Kohnstamm, Ueber retrograde Degeneration. Schmidt's Jahrb. d. ges. Med. Bd. CCLXI. 1899.

11) Derselbe, Ueber die gekreuzt aufsteigende Spinalbahn etc. Neurol.Cbl.1900. 6.

12) Derselbe, Ueber die Coordinationskerne des Hirnstamms etc. Monatsschr. f. Psych. u. Neurol. 1900.

13) Derselbe, Zur Theorie der Reflexe von hinterer Wurzel auf hintere Wurzel. Cbl. f. Phys. 1900. 18.

14) W. Kolmer, Beitr. zur Kenntniss d. mot. Hirnrindenregion. Arch. f. mikrosk. Anat. 1899. IX, 2.

15) Langendorff, Sitzungen d. naturf. Gesellschaft zu Rostock. 1898. 5.

16) Mislawsky, Cbl. f. Physiol. 1900. 9.

17) Sherrington, On the question, whether any fibres of the mammalian dorsal (afferent) spinal root are of intraspinal origin. Journ. of physiol. Vol. XXI.

1S) Steinach und Wiener, Pff. Arch. f. d. ges. Phys. LX. 1845.

19) E. Stein a ch, Ueber die viscero-mot. Function d. hint. Wurzeln etc. Pfl. Arch. f. d. ges. Phys. LXXI. 1898.

20) Derselbe, Ueber die centripet. Erregungsleitung im Bereiche der Spinalganglien. PA. Arch. f. d. ges. Phys. LXXVIII. 1899.

21) Stricker, Sitzber. d. k. Ak. d. Wiss. Wien LXXIV. Abth. III. 1876 und Vorlesungen über allgem. u. exper. Pathologie. Wien 1877. S. 668.

22) A. Wallenberg, Das mediale Opticusbündel. Neurol. Cbl. 1898. 12. 